\title{
Planned home birth: benefits, risks, and opportunities
}

\author{
This article was published in the following Dove Press journal: \\ International Journal of Women's Health \\ 8 April 2015 \\ Number of times this article has been viewed
}

\author{
Ruth Zielinski' \\ Kelly Ackerson ${ }^{2}$ \\ Lisa Kane Low ${ }^{1,3,4}$ \\ 'School of Nursing, University \\ of Michigan, Ann Arbor, MI, USA; \\ ${ }^{2}$ Bronson School of Nursing, Western \\ Michigan University, Kalamazoo, \\ MI, USA; ${ }^{3}$ Department of Obstetrics \\ and Gynecology, Medical School, \\ University of Michigan, Ann Arbor, \\ MI, USA; ${ }^{4}$ Women's Studies \\ Department, University of Michigan, \\ Ann Arbor, MI, USA
}

\begin{abstract}
While the number of women in developed countries who plan a home birth is low, the number has increased over the past decade in the US, and there is evidence that more women would choose this option if it were readily available. Rates of planned home birth range from $0.1 \%$ in Sweden to $20 \%$ in the Netherlands, where home birth has always been an integrated part of the maternity system. Benefits of planned home birth include lower rates of maternal morbidity, such as postpartum hemorrhage, and perineal lacerations, and lower rates of interventions such as episiotomy, instrumental vaginal birth, and cesarean birth. Women who have a planned home birth have high rates of satisfaction related to home being a more comfortable environment and feeling more in control of the experience. While maternal outcomes related to planned birth at home have been consistently positive within the literature, reported neonatal outcomes during planned home birth are more variable. While the majority of investigations of planned home birth compared with hospital birth have found no difference in intrapartum fetal deaths, neonatal deaths, low Apgar scores, or admission to the neonatal intensive care unit, there have been reports in the US, as well as a meta-analysis, that indicated more adverse neonatal outcomes associated with home birth. There are multiple challenges associated with research designs focused on planned home birth, in part because conducting randomized controlled trials is not feasible. This report will review current research studies published between 2004 and 2014 related to maternal and neonatal outcomes of planned home birth, and discuss strengths, limitations, and opportunities regarding planned home birth.
\end{abstract}

Keywords: home birth, hospital birth, risks, benefits, maternal satisfaction

\section{Background}

For women in most developed nations, the choice of where to give birth is not really a consideration, because birthing in a hospital is the cultural norm. The hospital is where their mothers and their grandmothers most likely gave birth. This is, however, a relatively recent phenomenon. While time parameters vary from country to country, most developed countries experienced a dramatic shift from home to hospital birth during the 20 th century. In the UK, for example, $80 \%$ of women gave birth at home in the $1920 \mathrm{~s}$, and in 2011 only $2.3 \%$ of births occurred at home. ${ }^{1}$ The US had a similar shift, from 50\% home births in 1938 to fewer than 1\% in $1955 .^{2}$ New Zealand has slightly higher rates of home birth, at $2.5 \%,{ }^{3}$ and in the Netherlands approximately $20 \%{ }^{4}$ of births still occur at home. However, while rates of home birth remain low (Table 1), ${ }^{1-10}$ there is evidence of a small but significant increase in home birth rates in some countries. In the UK, rates of home birth increased from 1\% in 1991 to $2.3 \%$ in $2012 .{ }^{1}$ In the US, the planned home birth rate increased to $0.89 \%$ in 2012 (up from $0.56 \%$ in 2004). ${ }^{2}$ Whether or not this subtle shift in home birth rates will continue remains to be seen.
Correspondence: Ruth Zielinski

School of Nursing, University of Michigan,

Room 3336 SON, $400 \mathrm{~N}$ Ingalls, Ann

Arbor, MI 48109-5482, USA

Tel +l 7346470324

Fax +I 7349365525

Email ruthcnm@umich.edu 
Table I Comparison of home birth rates by country

\begin{tabular}{|c|c|c|}
\hline Country & Year/s & Rate \\
\hline England and Wales' & 2012 & $2.3 \%$ \\
\hline Sweden ${ }^{5}$ & |992-200| & $0.1 \%$ \\
\hline US $^{2}$ & 2012 & $0.89 \%$ \\
\hline Japan ${ }^{6}$ & 2010 & $1.1 \%$ \\
\hline Finland ${ }^{7}$ & 2012 & $0.6 \%$ \\
\hline the Netherlands ${ }^{4}$ & 2013 & $20 \%$ \\
\hline Canada $^{8}$ & 2008 & $1.2 \%$ \\
\hline Australia ${ }^{9}$ & 2011 & $0.4 \%$ \\
\hline New Zealand ${ }^{3}$ & 2011 & $3.3 \%$ \\
\hline Norway ${ }^{10}$ & 1990-2007 & $0.8 \%$ \\
\hline
\end{tabular}

Increased attention to the issue of home birth in recent years is evident in the media and in research, as well as by professional organizations. In the UK, the Royal College of Midwives and the Royal College of Obstetricians and Gynaecologists issued a joint statement that "support(s) home birth for women with uncomplicated pregnancies." ${ }^{11}$ A recent publication from the National Institute for Health and Care Excellence in the UK states that low-risk multiparous women should be advised that birthing at home is as safe for the baby and that the rate of interventions for them will be lower than in the hospital setting. ${ }^{12}$

Position statements issued by maternity care organizations in the US illustrate the differing viewpoints and demonstrate the variance in interpretations of the outcomes of planned home birth. While the American College of NurseMidwives $^{13}$ and the Midwives Alliance of North America ${ }^{14}$ support informed choice and access to home birth, the American Congress of Obstetricians and Gynecologists ${ }^{15}$ and the American Academy of Pediatrics ${ }^{16}$ maintain that hospitals or birthing centers are the safest place for women to birth, regardless of risk status.

Within the debate surrounding home birth, the emphasis is often placed on infant outcomes, reported as stillbirths, neonatal deaths, or Apgar scores. While infant outcomes are vital to the safety of home birth, maternal mortality and morbidity are also important and less often mentioned in these debates. Even less frequently are issues of maternal satisfaction with the birth experience considered. The focus of this review is to explore the issue of risk and benefits to both mother and infant related to place of birth - specifically home birth.

\section{Methods}

Because the issue of childbirth risk and access to health care facilities is very different in developing countries, this exploration is limited to those studies undertaken in developed countries. We chose to include primarily studies that had been conducted within the last 10 years, to avoid questions of advancing resources or changing risk factors over time with investigations conducted prior to the last decade. Additionally, there is literature that addresses the safety and care of women in birth center settings compared with hospitals that will not be included, as the focus of this review is home compared with hospital for the place of birth. Both quantitative and qualitative study findings were included, as the intent was to report not only the safety data related to home birth but also psychosocial elements such as satisfaction with the birth experience.

\section{Search strategy}

Primary research studies, meta-analyses, and opinion papers were identified by searching electronic databases and reviewing reference lists. With exceptions for landmark publications, we included only those published in the last 10 years. Search engines included PubMed, CINAHL, and ProQuest. Search terms included "homebirth", "home birth", and "out of hospital birth", with the majority of relevant findings yielded using "homebirth". We limited the search to publications in English, and within ProQuest we narrowed the search to "scholarly journals only". The electronic searches yielded 164 publications using PubMed, 202 for CINAHL, and 298 for ProQuest. Two annotated bibliographies of home birth outcomes were an additional resource for pertinent articles utilized in this review. ${ }^{17,18}$ The authors reviewed each title and abstract for relevance to home birth, excluding those that were not relevant. A total of 23 primary quantitative reports and nine qualitative study reports were included in this review. Of the 23 quantitative reports, for 21 the primary outcome variables were related to safety (Table 2). Two quantitative reports and all nine qualitative studies were related to maternal satisfaction or motivation for choosing home birth (Table 3). Of note, while the search included studies published within the last 10 years, the majority of publications were within the last 5 years. Additional publications related to home birth, such as guidelines, protocols, and birth data reports, were also utilized for this review.

\section{Results}

\section{Challenges with researching home birth}

The "gold standard" for researching clinical outcomes remains the randomized controlled trial (RCT). Indeed, when comparing home birth with hospital, women who choose to birth at home are demographically different from the overall population of childbearing women. Birth certificate data in 


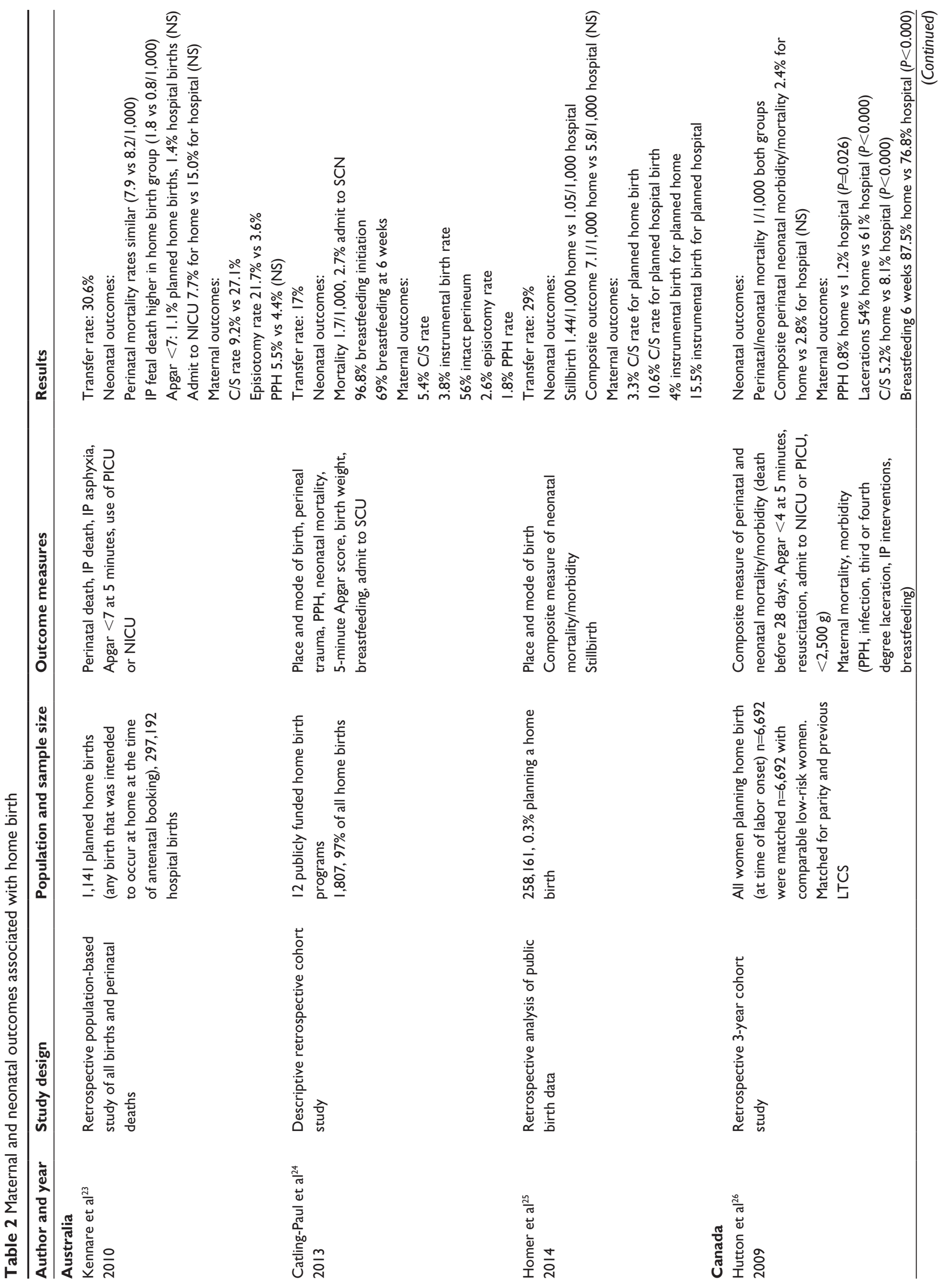




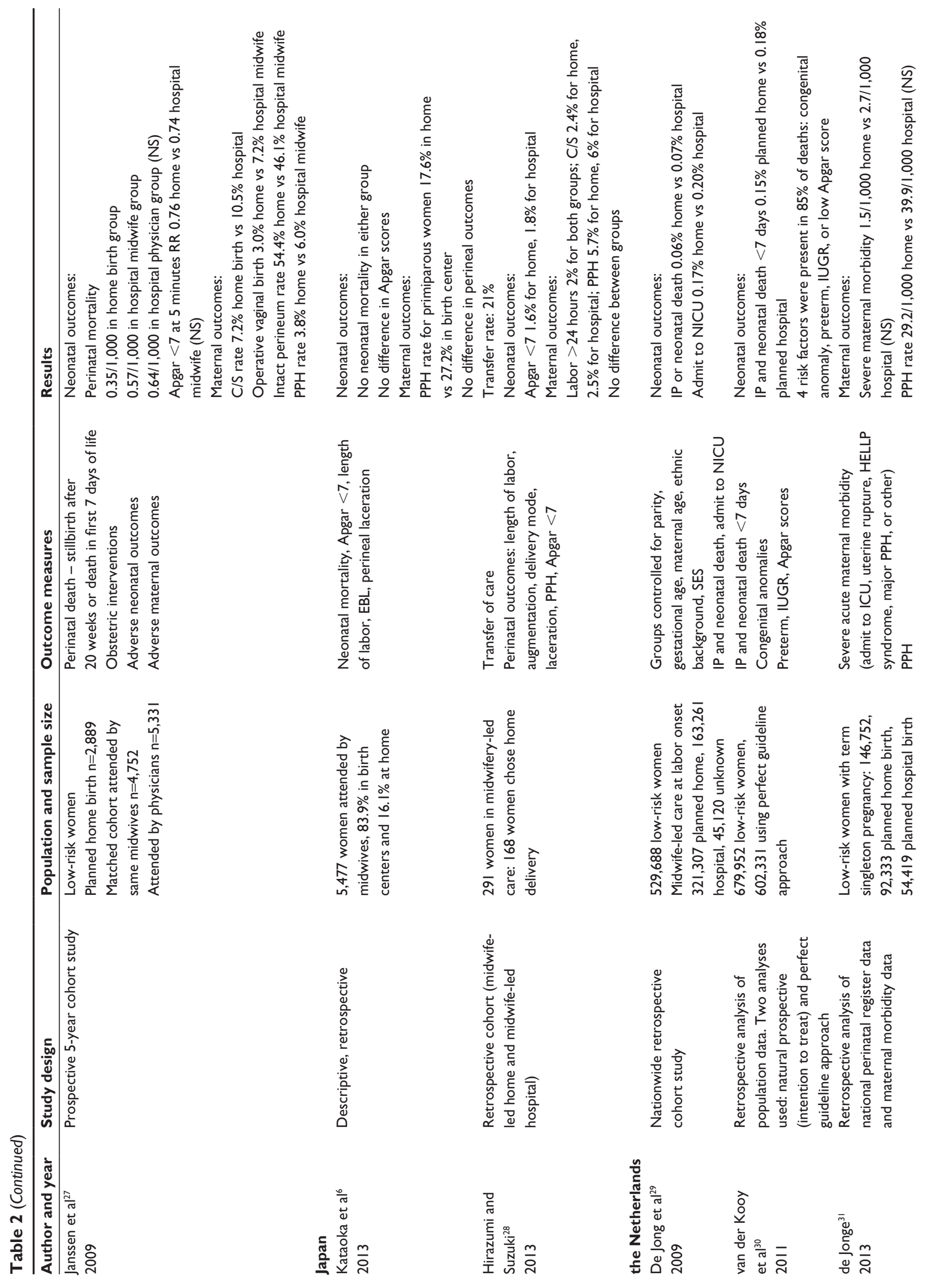




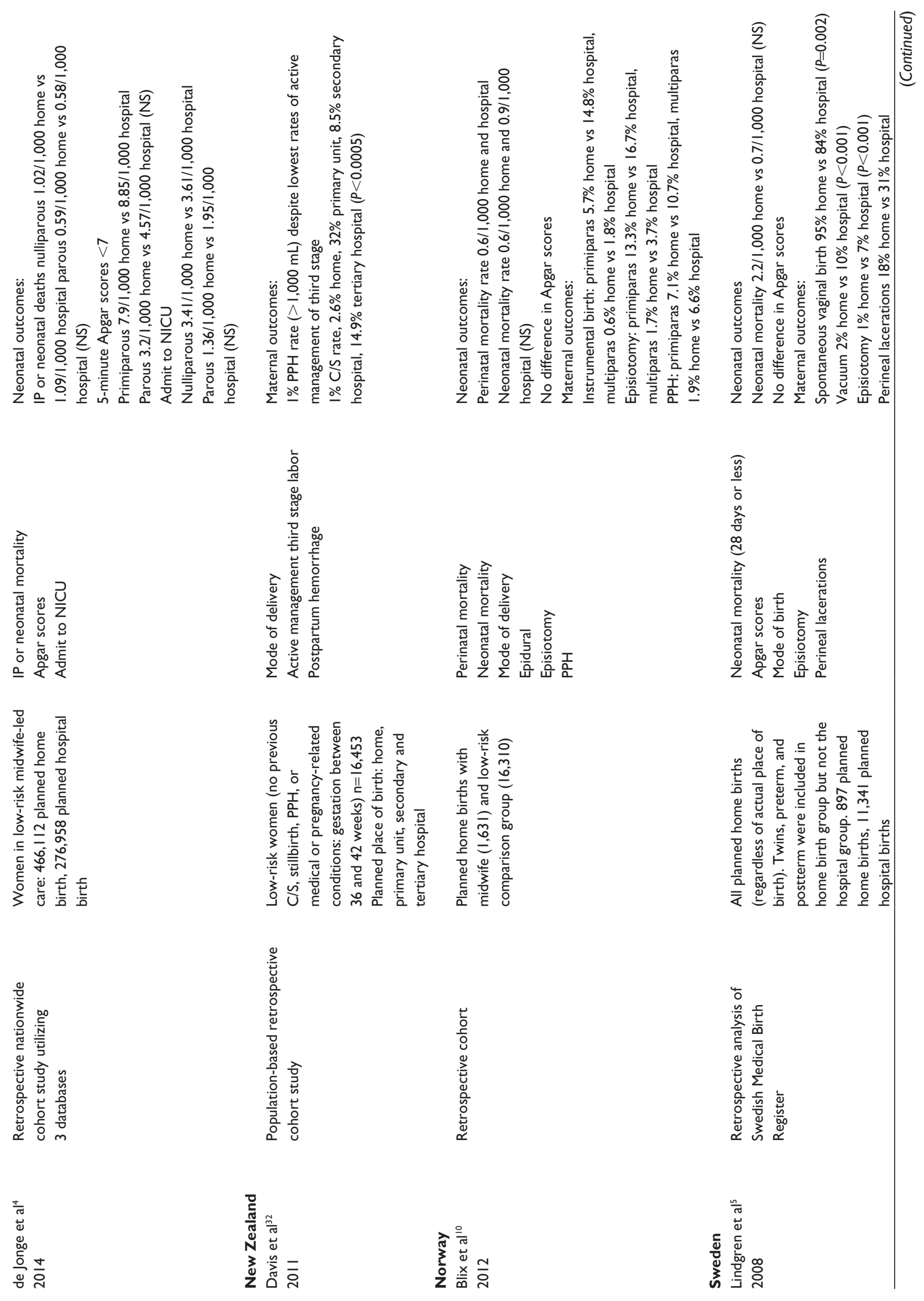




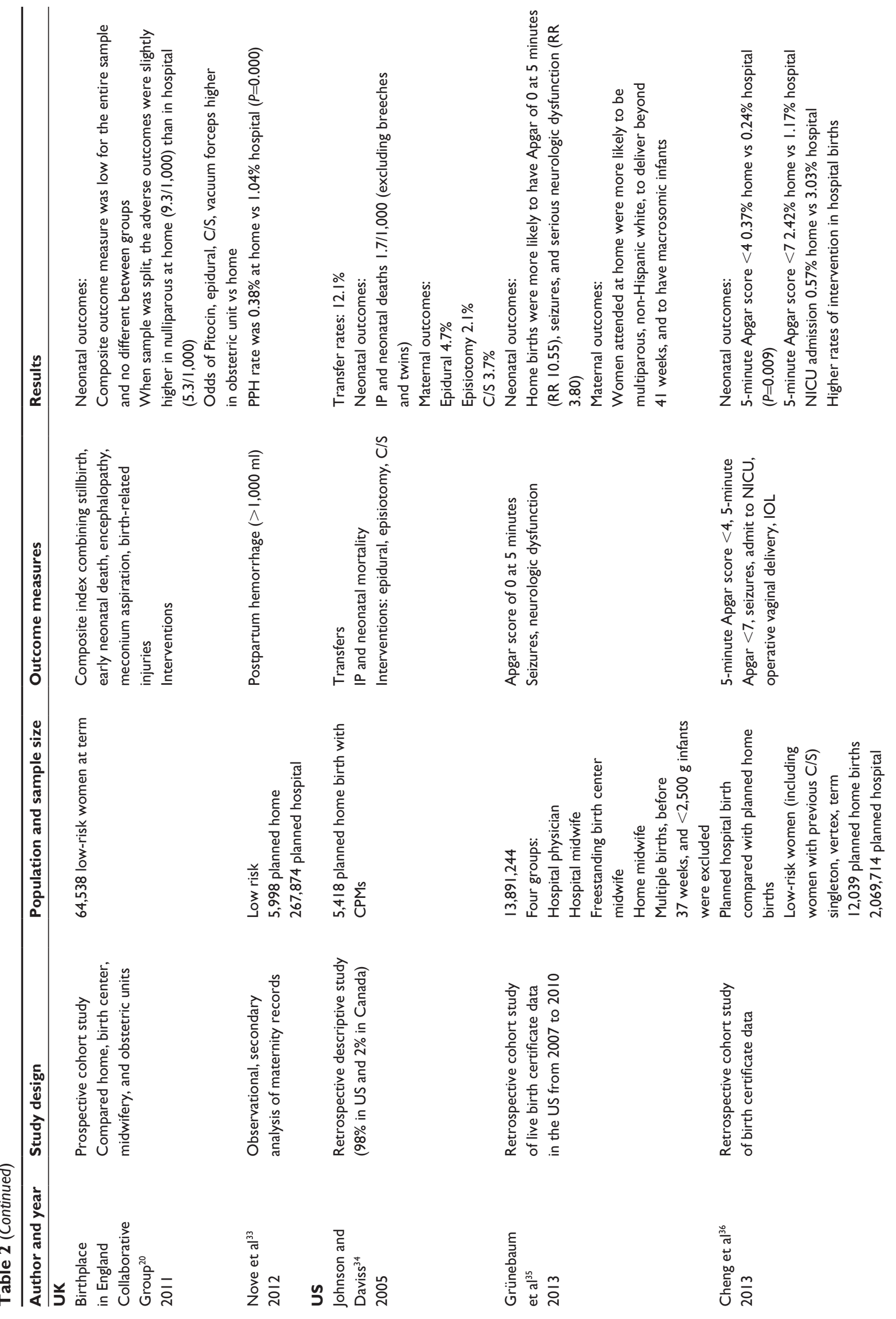



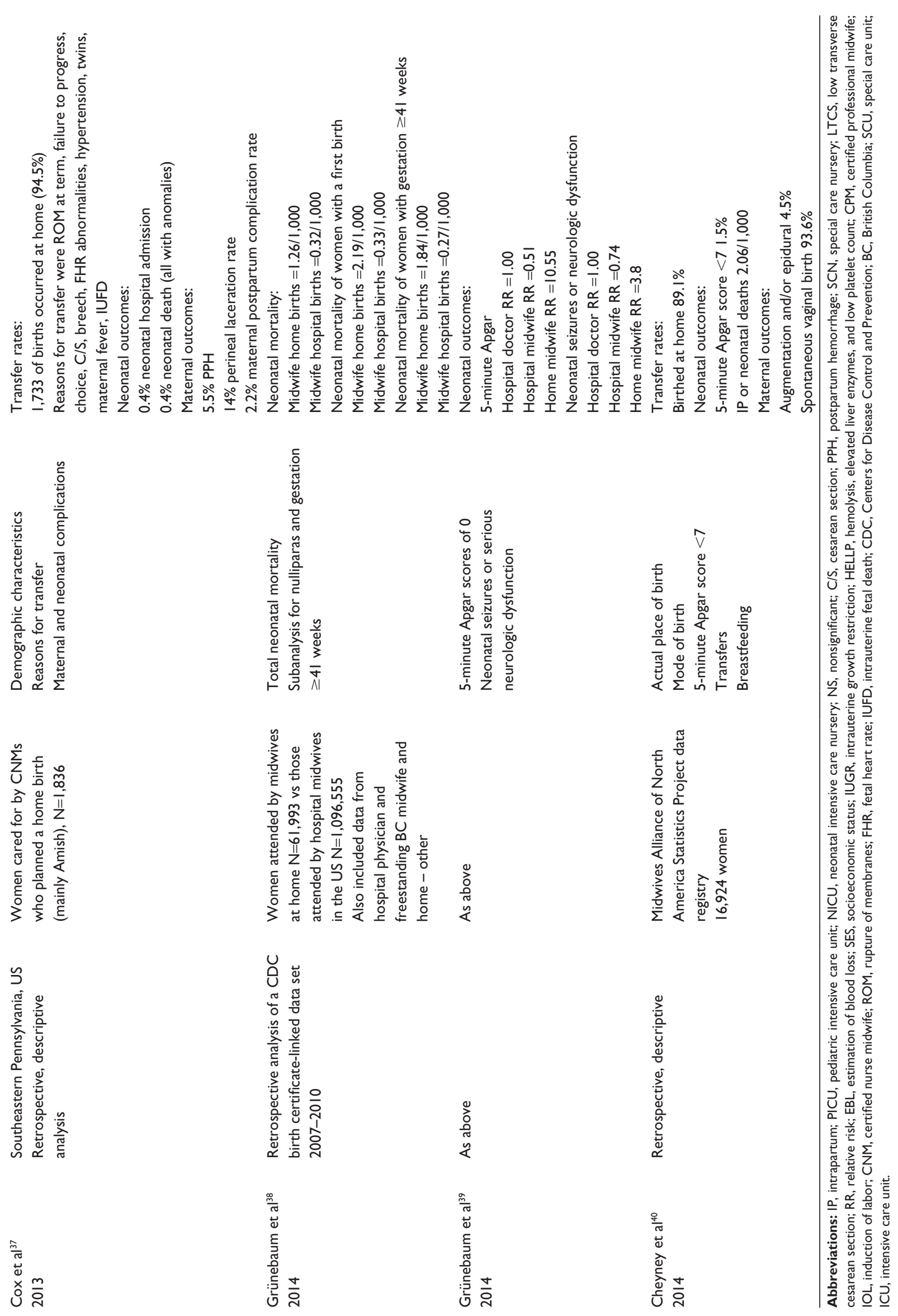


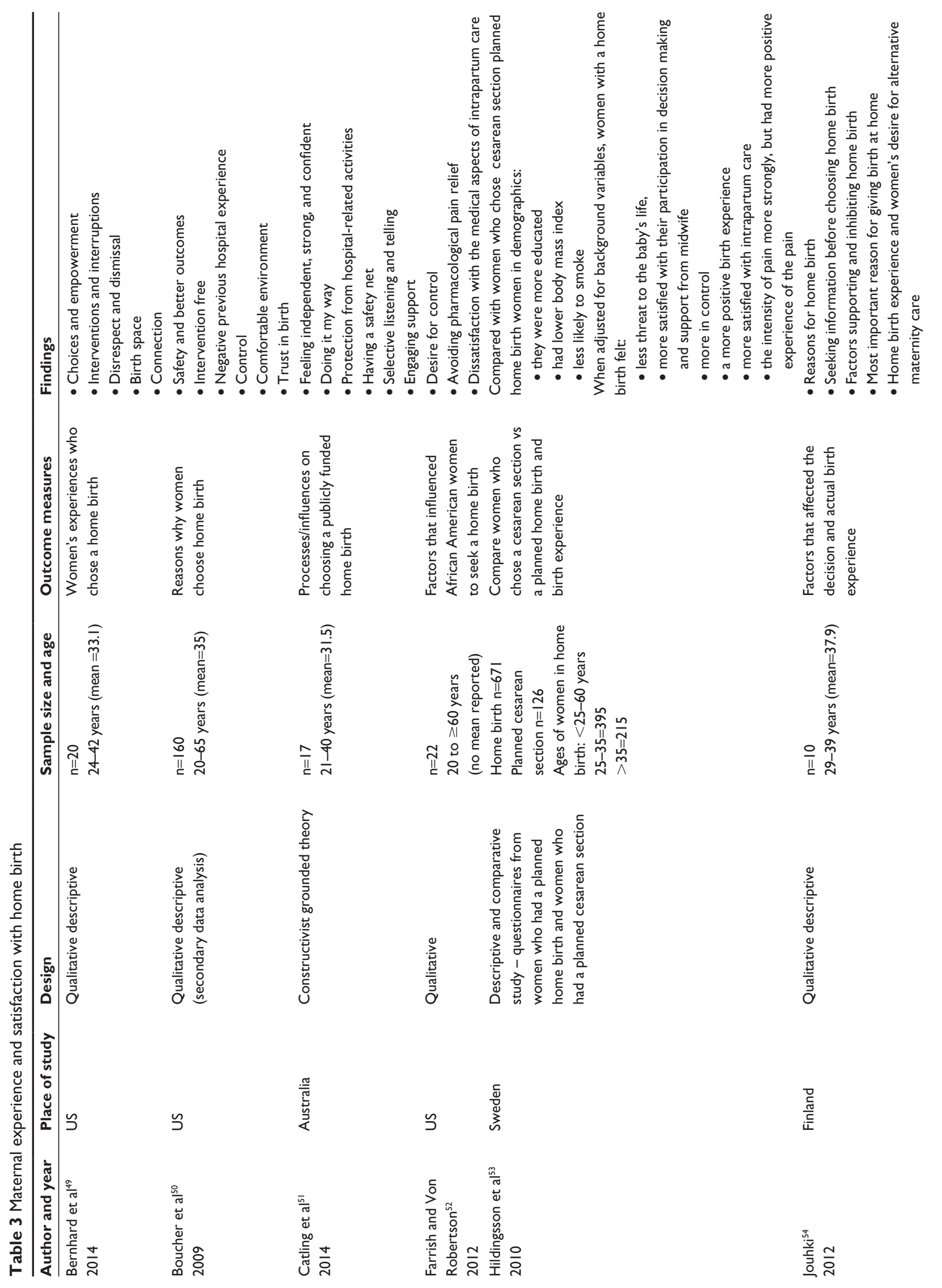



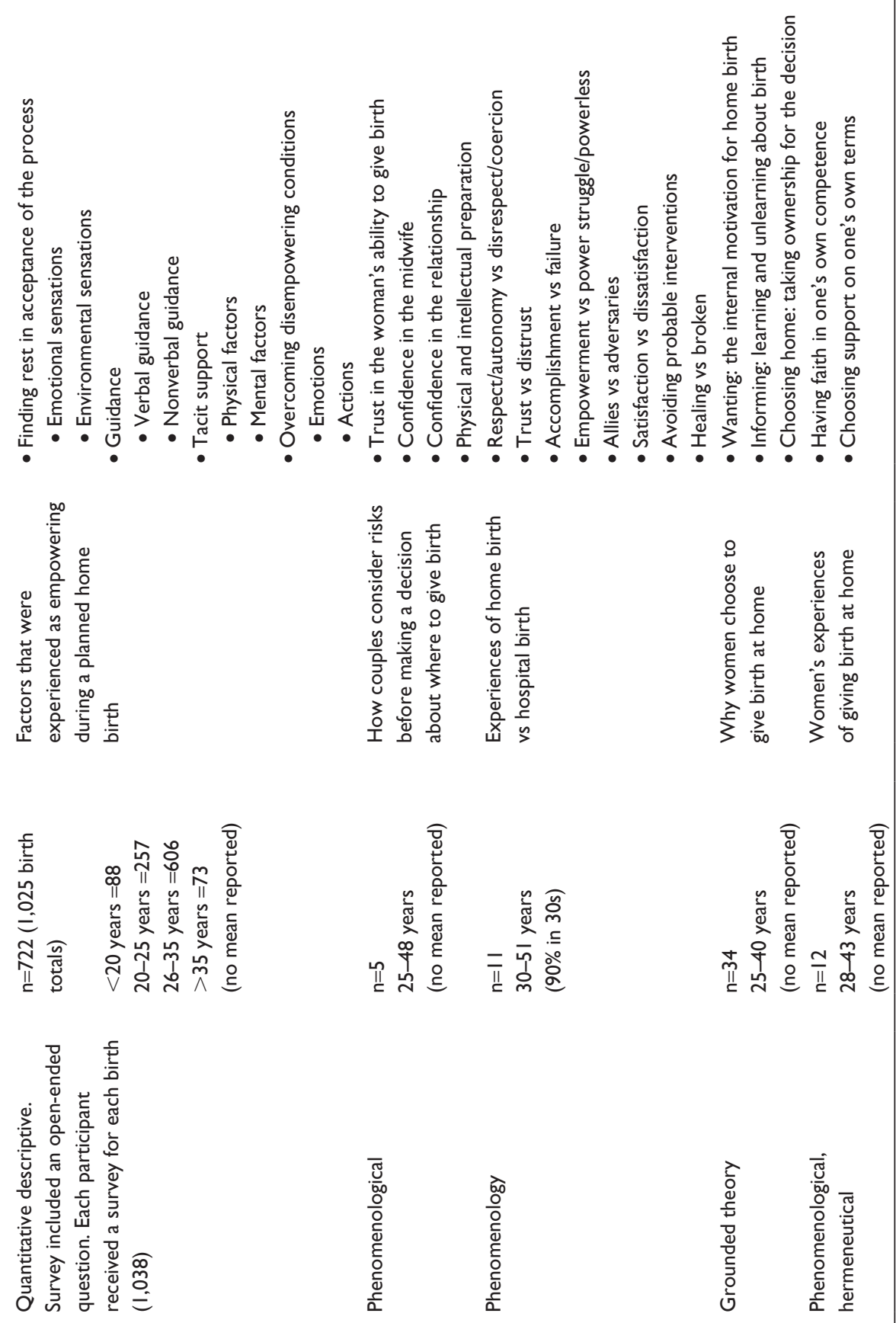

㐫

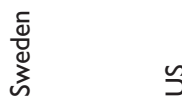

U
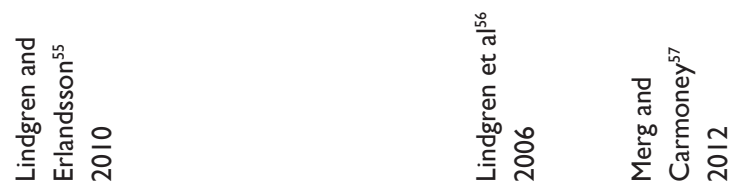

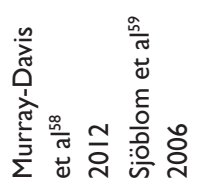


the US indicate that women who have a planned home birth are more likely to be white, married, and not having their first baby. ${ }^{2}$ Data from Sweden indicate that women choosing home birth there are more likely to have larger families, be older, have a higher level of education, and not work outside the home. ${ }^{19}$ Women choosing out of hospital birth in the UK are more likely to be older, white, and of higher socioeconomic status. ${ }^{20}$ Women in the Netherlands who plan a home birth are more likely to be older, multiparous, of Dutch origin, and have a medium or high socioeconomic status. ${ }^{4}$

To date, there have been two attempts to conduct an RCT of home birth. The first, in 1996, was conducted in the UK. ${ }^{21}$ In this feasibility study, eleven participants were recruited from 71 women who met the criteria for a home birth. While the authors suggest that a larger trial might be possible, they acknowledge that because of the low incidence of severe morbidity or mortality, safety as an outcome variable would not be feasible in an RCT of home birth. ${ }^{21}$ In 2009, researchers in the Netherlands, where home birth rates are the highest, designed an RCT and were able to enroll only one participant after 6 months of recruitment. ${ }^{22}$ The study was then redesigned to explore reasons why women are reluctant to enroll in a home birth trial. ${ }^{22}$ The main reasons women declined participation were that either they had already decided where they wanted to give birth or they wished to choose their own place of birth. ${ }^{22}$ Since participation in research must be voluntary, it is clear that RCTs of place of birth are not feasible. Therefore, studies of home birth outcomes must rely on observational methods.

Outcomes from large observational studies in various countries have been conducted (Table 2)..$^{4-6,10,20,23-40}$ In addition, a number of systematic reviews of the findings have been published, ${ }^{39,40}$ as well as a meta-analysis of prior data. ${ }^{41}$ Some prospective studies utilize an "intention to treat" whether early in pregnancy ${ }^{23}$ or at the onset of labor, ${ }^{20}$ while others compare outcomes based on where women ultimately birthed. ${ }^{35,36}$ Utilizing an "intention to treat" model may artificially increase the adverse outcomes in the home birth group when women are appropriately transferred due to risk factors. However, using ultimate birth location may result in an artificially low rate of interventions such as cesarean section, because women would appropriately be transferred prior to these interventions.

A challenge associated with comparison of home birth findings is that the studies are conducted in different countries with a variety of home birth providers. For example, while two studies undertaken in Canada compared home versus hospital outcomes of births undertaken by the same midwives, ${ }^{26,27}$ other studies compare outcomes with differing birth attendants (physicians, midwives, nurse--midwives, licensed, nonlicensed). ${ }^{35,36}$

An additional challenge is the population included in the home birth data. Unplanned home birth often includes women and infants for whom there is greater risk of mortality/ morbidity (prematurity, no prenatal care). ${ }^{41}$ For this reason, most current studies include only planned home births. However, when studies in the US utilize birth certificate data, unplanned home births may inadvertently be reported, because some states do not distinguish between planned and unplanned home births. ${ }^{2}$

\section{Neonatal outcomes}

When investigations of safety during planned home birth are conducted, neonatal outcome variables have been analyzed and reported in a variety of ways. Because serious morbidity and mortality are infrequent occurrences, some studies utilize a composite index that combines, for example, rates of intrapartum stillbirth, neonatal encephalopathy, and birth-related injuries. ${ }^{21}$ The strength of utilizing this design is the power to determine differences for outcomes that are relatively rare. However, this approach assigns the same degree of importance to an outcome such as an intrapartum stillbirth and a fractured clavicle, which is a relatively benign birth injury. Other studies report neonatal deaths, neonatal intensive care (NICU) admissions, and 5-minute Apgar scores. Neonatal deaths are sometimes reported as early neonatal deaths ( $\leq 7$ days) or neonatal deaths ( $\leq 28$ days), adding to challenges in analysis and interpretation of findings. Five-minute Apgar scores are also reported in a variety of ways $-<7,<4$, or $0-$ which, similarly, makes analysis and interpretation of research findings more challenging.

Studies conducted regarding neonatal outcomes related to home birth have reported conflicting results across various countries and populations of women. Some studies conducted in the US suggest that there may be an increased frequency of low Apgar scores and adverse neurologic outcomes in infants born at home in comparison with hospital. ${ }^{35,36,38,39}$ In a retrospective descriptive cohort study, infants born at home were more likely to have a 5-minute Apgar score of 0, neonatal seizures, or serious neurologic dysfunction. ${ }^{35}$ Data for this study were collected from the National Center for Health Statistics birth certificate data and included information from all birth certificates from 2007 to $2010 .{ }^{35}$ Preterm and multiple gestations were excluded. Apgar scores were included on all birth certificates; however, other neonatal outcomes were incomplete because these data were not consistently 
collected in all 50 states. While the analysis was comprehensive, the utility of birth certificate data for determining safety of home birth is limited. For example, a 5-minute Apgar score of 0 does not determine whether events occurred during the antepartum or intrapartum period. Why an Apgar score of 0 versus a more clinically significant measure such as $<7$ at 5 minutes $^{42}$ was analyzed is not clear. Additionally, this analysis did not differentiate between types of midwife attending the birth (lay midwife, certified professional midwife, certified midwife, or certified nurse-midwife). While the risk of adverse neonatal outcomes was not analyzed by specific risk factors, in a separate publication the authors report that there were more women with risk criteria (breech presentation, prior cesarean, twins, or gestational age over 41 weeks) in those attended by home birth midwives compared with the nurse-midwife-attended hospital births. ${ }^{38} \mathrm{~A}$ later publication by the same authors utilizing a Centers for Disease Control and Prevention birth/infant death data set from 2006 to 2009 indicated a higher total neonatal mortality risk in infants born at home (1.26 vs 0.32 per 1,000$).{ }^{39}$ Risk of adverse neonatal outcomes increased if gestation exceeded 41 weeks or if it was the mother's first birth. ${ }^{39}$

An additional retrospective study done in the US utilizing birth certificate data compared women who had planned home births versus planned hospital births. ${ }^{36}$ Only women having term singleton vertex births were included; however, previous cesarean births were not excluded. For this study, Apgar score $<4$ was the primary outcome, with secondary outcomes of Apgar score $<7$, NICU admission, and obstetric interventions. The authors report an increase in 5-minute Apgar scores $<4(0.37 \%$ vs $0.24 \%)$, higher rates of neonatal seizures $(0.06 \%$ vs $0.02 \%)$ with no difference in assisted ventilation rates, and lower rates of NICU admissions among infants born at home. ${ }^{36}$ When a subanalysis was undertaken by midwife type, there was no difference in Apgar score between home and hospital among births attended by certified nurse-midwives, and the only difference that remained was that neonates born at home had fewer NICU admissions than those born in the hospital. ${ }^{36}$ While birth certificate and associated data sets provide opportunity for large amounts of data, the reliability of some variables, such as labor and birth complications and congenital anomalies, has been brought into question. ${ }^{43}$

In a descriptive analysis of planned home birth among primarily Amish women in Pennsylvania, US, the rate of neonatal death was $0.4 \%$, and all were attributed to fetal anomalies. ${ }^{37}$ The rate of transfer to the hospital was also low $(0.75 \%)$, despite many women having a high parity. ${ }^{37}$
Of note, in this study, the birth attendants were certified nurse-midwives, and practice guidelines required that women were low risk and the midwives reported a good working relationship with the transferring facilities. ${ }^{37}$ Also, because the population was primarily Amish, where birth at home is normalized, generalizability to the rest of the population is limited. ${ }^{37}$ However, similar descriptive studies report similarly low neonatal mortality rates ( 1.7 per $1,000)$ when breech and twin home births were excluded and the reported Apgar score of $<7$ rate was $1.3 \% .{ }^{34} \mathrm{An}$ additional descriptive study was undertaken analyzing both planned and actual rates of recent (2004-10) home birth data from the Midwives Alliance of North America database. ${ }^{40}$ Low Apgar scores ( $<7$ at 5 minutes) occurred in 1.5\% of newborns, with $0.6 \%$ having a 5-minute Apgar score $<7 .{ }^{40}$ When infants with lethal anomalies were excluded, the rate of intrapartum fetal death was 1.30/1,000, the rate of early neonatal death was $0.41 / 1,000$, and the rate of late neonatal death was $0.35 / 1,000 .{ }^{40}$ Infants born in breech position were at significantly increased risk for death at all time points (13.51/1,000, 4.57/1,000, and 4.59/1,000), while infants born to primiparous women or those with a prior cesarean section were increased only at the intrapartum time point $(2.91 / 1,000$ and 2.85/1,000, respectively). ${ }^{40}$ When higher-risk women were excluded from the analysis, the intrapartum death rate decreased to $0.85 / 1,000 .{ }^{40}$ Of note, the data in this sample were obtained from midwives who voluntarily participated in data collection and self-reported the outcomes. ${ }^{40}$

Three studies of neonatal outcomes of planned home birth have been undertaken in the past 10 years in Australia. ${ }^{23-25}$ In South Australia, perinatal data for all births from 1991 to 2006 were analyzed, including data from 1,141 planned home births and 297,192 hospital births. ${ }^{23}$ Planned home births were defined as births that were intended to occur at home at the time of the first prenatal visit; of these, $30.6 \%$ occurred in the hospital. ${ }^{23}$ The perinatal mortality rate for home birth was similar to rates within the hospital; however, the authors report that there were three planned home birth infants who died due to intrapartum asphyxia, concluding that this was a higher number than expected..$^{23}$ In this study, Apgar scores of infants transferred to the hospital from a planned home birth were lower than Apgar scores of planned hospital births. ${ }^{23}$ This is not unexpected, since transfers represent women at higher risk of untoward outcomes, and in the US, barriers to transfer exist in the current maternity care system. ${ }^{44}$ In New South Wales, Australia, using a variety of routinely collected linked data, the rates of stillbirth and neonatal deaths were not significantly different between 
hospital $(5.8 / 1,000)$, home $(7.1 / 1,000)$, and birth center $(5.3 / 1,000)$, although the authors report that the study was underpowered to achieve statistical significance. ${ }^{25} \mathrm{~A}$ descriptive study of publicly funded home birth in Australia reported a stillbirth and early neonatal death rate of 3.3/1,000, which was reduced to $1.7 / 1,000$ when deaths related to fetal anomalies were excluded. ${ }^{24}$

Safety of home birth in relation to neonatal outcomes was explored using a prospective design in British Columbia, Canada. ${ }^{26} \mathrm{~A}$ strength of this study design was that the same providers (registered midwives) attended both the home births and the comparison hospital group. ${ }^{26}$ Planned place of birth determined group placement versus actual place of birth. There were no differences in rates of perinatal deaths, which were low among all groups (home birth $=0.35 / 1,000$ and $0.57 / 1,000$ for hospital midwife attended). ${ }^{26}$ Neonatal adverse outcomes such as rates of birth trauma, resuscitation, and meconium aspiration were similarly low for both groups, with no increase in adverse outcomes for planned home births. ${ }^{26}$

Utilizing a composite index of perinatal mortality and morbidity, infants born outside the hospital (home and freestanding or alongside midwifery units) in the UK were no more likely to have an adverse event than those born within the hospital. ${ }^{20}$ For this study, the researchers utilized an intention-to-treat model based on planned place of birth at the onset of labor. ${ }^{20}$ All women were healthy and low risk. However, in a subgroup analysis by parity, nulliparous women in the planned home birth group were more likely to have an adverse neonatal outcome than those planning hospital birth. ${ }^{20}$

Studies conducted in the Netherlands, where home birth rates are the highest in the developed world, have not demonstrated an increase in adverse outcomes for infants during planned home birth. ${ }^{4,29,30}$ In a retrospective analysis, records from 679,952 low-risk women were investigated to compare intrapartum and early neonatal mortality rates for low-risk women during planned home birth and hospital birth with a midwife. ${ }^{30}$ The risk of intrapartum or early neonatal death was $0.15 \%$ for home birth versus $0.18 \%$ for hospital birth, a difference that did not reach statistical significance when preterm births ( $<36$ weeks) were excluded. ${ }^{30}$ In certain subgroups of women (very young or old or with small for gestation age), the risk may be slightly increased. ${ }^{30}$ Similarly, two additional nationwide cohort studies in the Netherlands of low-risk women attended by midwives demonstrated no increased neonatal adverse outcomes (death or NICU admission) associated with home birth. ${ }^{4,29}$
A meta-analysis undertaken and published in 2010 included data from 12 studies and a total of 342,056 planned home and 207,551 planned hospital births. ${ }^{45}$ The authors report that in the home birth group, gestation was significantly more likely to exceed 42 weeks. While the perinatal mortality rate was similar for both groups, the overall neonatal death rate was higher in the planned home birth group $(0.20 \%)$ versus the planned hospital group $(0.09 \%) .{ }^{45}$ When deaths from fetal anomalies were excluded, the rates were $0.15 \%$ for home birth versus $0.04 \%$ for hospital. ${ }^{45}$ While the benefit of combining data to increase the power to detect significance is compelling, comparing studies from differing countries across a 30-year timespan (1976-2006) and with varied data collection methods is of questionable value. ${ }^{46}$ Notably, a majority of the births included in this study were taken from studies in the Netherlands that did not find a similar difference in mortality in the primary analysis..$^{29,30}$ While the meta-analysis reports the comparison as being between planned home birth and planned hospital birth, one included study used data obtained from birth certificates that did not differentiate between planned and unplanned home births. ${ }^{47}$ Finally, one of the largest studies that was initially included in the meta-analysis for some of the outcomes but had not yet published neonatal morbidity and mortality rates out to 28 days has subsequently provided this information. The findings of this cohort investigation of 743,070 low-risk planned home and hospital births in the Netherlands found no increased risk of adverse neonatal outcomes with planned home birth when extending the time period out to 28 days for neonatal outcomes. ${ }^{4}$ As a result, the authors of this publication suggest that if their results had been included in the meta-analysis conducted by Wax et al, ${ }^{45}$

\footnotetext{
their conclusion inevitably would have been that not only perinatal mortality but also neonatal mortality was similar for planned home births versus planned hospital births, as more than $95 \%$ of home births in their study came from our data. ${ }^{4}$
}

\section{Breastfeeding}

Breastfeeding rates were included as a variable in two of the studies reviewed. In a Canadian study, infants born to women planning home birth were significantly more likely to be exclusively breastfeeding 1 week postbirth (91.5\%) than those born during planned hospital birth $(84.5 \%) .{ }^{26}$ This difference remained at 6 weeks postpartum, with $87.5 \%$ of infants being exclusively breastfed in the home birth group (vs 76.8\% in the hospital birth group). ${ }^{26}$ While groups in this 
study were matched on demographic characteristics such as age and parity, there may well be other factors that motivate women to plan a home birth and also exclusively breastfeed. Similar rates were reported among infants born at home in the US, with $97.9 \%$ at least partially breastfeeding and $86 \%$ exclusively breastfeeding at 6 weeks postpartum. ${ }^{40}$

\section{Maternal outcomes}

Women who have a planned home birth experience fewer interventions such as operative vaginal delivery and induction of labor when compared with women who birth within a hospital setting. ${ }^{36}$ This finding is not surprising, since interventions such as operative vaginal birth or induction of labor with medication are typically not undertaken at home; rather, transfer to the hospital would be done prior to these interventions. A more realistic comparison can be made using an "intention to treat" model where participants are grouped based on their plan to have a home birth at time of labor onset, allowing the opportunity to compare rates of intervention such as cesarean section. In a Canadian study that matched women by risk factors, parity, and history of a prior low transverse cesarean section, women who intended to birth at home had cesarean section rates of $5.2 \%$ versus $8.1 \%$ in the low-risk women who planned a hospital birth. ${ }^{26}$ Findings from descriptive studies indicate similarly low cesarean section rates of $3.7 \%$ in women planning a home birth. ${ }^{34}$

Across all studies, women intending to birth at home are significantly less likely to have other obstetric interventions such as epidural anesthesia, forceps, vacuum, augmentation of labor, or episiotomy, regardless of where they ultimately give birth. ${ }^{20,23,25,26}$ This is somewhat to be expected, since these women are considered lower risk; however, these differences remain when a comparison design is utilized where women in both groups (home and hospital) were low risk and the same midwives were attendants. ${ }^{20,26,37}$ In New Zealand, where the home birth rate is $3.3 \%$, a population-based retrospective study demonstrated that women planning a home birth had lower rates of all interventions, including cesarean section and instrumental birth. ${ }^{32}$

While interventions that are unnecessary should clearly be considered a negative outcome, what is not clear is when those interventions may in fact be necessary, potentially preventing other adverse outcomes such as neonatal mortality or low Apgar scores. That said, there is consensus that the overall rate of interventions such as cesarean section is substantially higher than necessary. ${ }^{48}$

Studies indicate that maternal complications related to birth, such as postpartum hemorrhage and third and fourth degree perineal lacerations, are lower in women who plan a home birth. ${ }^{26}$ For example, in primarily Amish women attended at home by certified nurse-midwives, the rate was $13 \%$ for any perineal laceration and $0.25 \%$ for third or fourth degree lacerations. ${ }^{37}$ Of note, this population had high parity; only $17.1 \%$ were nulliparous. However, in this population, rate of postpartum hemorrhage was also low (5.5\%), despite $33.4 \%$ of the women having high (5-13) parity. ${ }^{37}$ Rates of perineal lacerations were similarly low for planned home births in southern Australia (34.2\% first to second degree, 1.1\% third or fourth degree). ${ }^{24}$ Rates of other maternal complications such as retained placenta or endometritis were also low in this home birth population. ${ }^{24}$ In comparison with planned hospital birth, postpartum hemorrhage rates ( $>500 \mathrm{~mL}$ blood loss $)$ are either the same ${ }^{32}$ or lower in women who plan home birth, ${ }^{26,27}$ and they are less likely to receive blood transfusion. ${ }^{20}$

\section{Women's experiences with birth setting}

For the studies that explored why women chose a home birth, eleven research studies were evaluated that described influencing factors contributing to a woman choosing a home birth. Table 3 presents a summary of the eleven research studies (nine qualitative and two quantitative) $)^{49-59}$ and addresses the major themes that evolved from the participants' responses.

Results of synthesizing the research studies with women regarding home births generated three major themes. The major themes were previous experiences of hospital birth, control and empowerment, and home environment.

\section{Experiences of hospital birth}

There were events that occurred during the birth process and hospital stay that motivated women to choose a home birth in subsequent pregnancies. Two central factors found to influence women's decision to choose a home birth after a hospital birth were too many interventions and interruptions and a desire to avoid pharmacological pain relief.

Not all interventions performed on laboring women are needed in order to have a good birth outcome. Women believed that the birth process is natural, not a disease, and would proceed much smoother if there were not so many interventions..$^{52,54,55,58,59}$ Unfamiliar people walking in and out of their hospital room contributed to a loss of concentration, increasing not only discomfort with the labor process but emotional discomfort as well. ${ }^{51,55}$ Along with unfamiliar people walking in and out, women were dissatisfied with care received by their own health care providers. ${ }^{49,52,57}$ Women felt they were treated disrespectfully and that procedures were 
conducted with no explanation on the provider's part, ${ }^{49-52}$ which can lead to mistrust.

One would think that women have the choice to use or not to use pharmacological pain relief during labor. Pharmacological pain management such as an epidural or narcotics must first receive a woman's informed consent. However, there was not a process of informed consent as perceived by women in many of the studies and thus became a basis for seeking home birth. Feelings of losing control during the birth process and "giving in", even though they did not want medication, was a key theme..$^{49,51,52,54}$ There were women whose birth plan was to avoid pain medications during labor. However, at some time during the labor process, women felt they were no longer able to sustain their desires due to pressure from the medical staff. ${ }^{49,51}$ Not all women want to receive medications for pain, because they believe labor pain is a normal process of labor. ${ }^{52,53}$ Feelings of pressure to receive pain medications may have contributed to women thinking differently and provided a basis for seeking an alternative, to give birth at home. In addition, Lindgren and Erlandsson ${ }^{55}$ found in their study involving 722 women with a total of 1,025 births, of which $75 \%$ of those births were at home, that pain was rarely mentioned as a factor in home birth. This finding may suggest that women who birth at home may be better prepared or prepare differently in pain management, and possibly that their caregiver helps guide them in coping techniques. Knowing that pharmacological pain relief is not available, women most likely discover other ways to manage normal birth, which may increase women's sense of control and feelings of satisfaction and accomplishment.

Women believed they were not being given choices in the hospital setting. Some believed that their voices were not heard. ${ }^{49-52,57}$ Women described their feelings that interventions were done that they felt were unnecessary and getting pain medications they really did not want, but felt their choice was taken away. When women are excluded from the decision-making process and/or decisional control is taken away, this ultimately may lead to dissatisfaction. Therefore, based on hospital birth experiences, home birth was chosen for subsequent births where they had perceived they would have greater control and opportunity for empowerment.

\section{Control and empowerment}

Choosing to birth in one's own home where women can control their environment and do it their own way and on their own terms, avoiding unnecessary interventions and interruptions, was consistently described by the women as empowering. ${ }^{49,51,52,55,57,58}$ Being the decision maker in how a woman would want her birth to go or being a part of the decision-making team was described as being important. Women felt that they were competent to make their own decisions and believed in themselves. ${ }^{52,54-56}$ Women became informed about home birth, learning about not only the risks but also the benefits. ${ }^{54,56,58}$ One such benefit was experiencing birth in a home environment.

\section{Home environment}

For most people, home is a peaceful and restful place. When a person is at home, they have more control of events that occur and over the environment. Giving birth in their own home on their own terms in a comfortable environment was more satisfying. ${ }^{49,50,52,55,57}$ It was also believed that birth would happen more normally at home without all of the interventions usually performed at the hospital. ${ }^{49,50,54,58}$ In the hospital setting, it is a different environment and culture, and hospital staff have certain routines that can affect the birthing process. It is not uncommon to have different people walk in and out of a laboring woman's room. In one's own home, people who enter are invited guests and are usually people who will provide the woman with good support. ${ }^{51,54,55}$ Good support is a helpful strategy to help a woman cope with the process of labor.

\section{Discussion}

While some studies suggest a small but significant increase in neonatal death and adverse outcomes, ${ }^{23,35,36,38,39,45}$ the majority of studies across a variety of countries have shown no increase in neonatal morbidity and mortality for planned home birth. 4,6,10,24-30,37,40 Additionally, maternal outcomes are consistently better for planned home birth, including less intervention and fewer complications. , $^{5,10,20,23-28,31,32-35,37}$ Satisfaction with the birth experience is also high in the home birth setting. ${ }^{53,57}$

Emotion, debate, and controversy surrounding the topic of home birth are clearly present, with strong opinions both for and against. ${ }^{60}$ Websites and blogs have proliferated, some in favor of and others warning against the risks of home birth. The perspective of some US-based maternity care providers is that physicians have a moral obligation to actively discourage women against choosing to give birth at home. ${ }^{61}$ Others argue that the principle of autonomy and thus a woman's right to make informed choices should prevail. ${ }^{46}$ These differing ethical perspectives, framing the issue of planned home birth within the context of autonomy and/or beneficence with the fetus as the primary focus, creates tensions within the health care provider arena as professional organizations then position themselves within this debate. ${ }^{60}$ 
Safety of home birth is dependent on many factors. Provision for home birth is vastly different depending on location, even within countries. For example, the Birthplace in England Research Programme conducted a qualitative exploration of the organizational and professional factors that may impact the safety of the home birth setting. ${ }^{62}$ They found that experience and comfort level for providing home birth services varied among midwives, as well as the amount of support and infrastructure available to support home birth. In the US, licensure for midwives varies greatly from state to state; thus, educational, certification, and practice experience may also vary widely, including legislation in some states that makes it illegal to provide home birth services. The International Confederation of Midwives recommends that global standards for midwifery education be a minimum of 3 years' postsecondary school at an accredited institution. ${ }^{63}$ Standardized educational requirements to establish a minimum level of preparation have been argued to be an important aspect of assuring the level of preparation to attend a home birth. ${ }^{64}$

In the US and other countries, there is not a consistent mechanism for home birth attendants to consult or transfer care during either pregnancy or labor ${ }^{44}$ In fact, home birth midwives in the US identify many barriers to accessing hospital care for their home birth clients. ${ }^{44}$ Transfer rates to hospital from planned home birth range from $9.9 \%$ to $31.9 \%$ and are higher in areas where home birth is an integrated part of the maternity system. ${ }^{65} \mathrm{~A}$ long distance to the nearest hospital and difficulty or delay in seeking transfer due to lack of an integrated system of health care may contribute to the potential for adverse neonatal outcomes. Despite the range of legal and health system barriers, as well as varied educational preparation of midwives within the US, a recent comprehensive observational cohort study reported outcomes of women who chose a planned home birth had similar findings to other countries - low rates of interventions, similar patterns for transfer of care when necessary, and no increase in adverse neonatal outcomes. ${ }^{44}$

Uniform guidelines outlining eligibility and risk factors for home birth have been argued to be an essential component for safe home birth. ${ }^{66}$ Evidence suggests that when guidelines are implemented and adhered to and when home birth is reserved for lower-risk women, home birth outcomes are as good as, or better than, outcomes of similar women within the hospital birth setting. ${ }^{20,66}$ Conversely, when women with risk factors such as breech or multiple birth have a home birth, there may be an increase in neonatal mortality and morbidity. ${ }^{39}$ Countries such as the Netherlands, ${ }^{67}$ New Zealand, ${ }^{68}$ and the $\mathrm{UK}^{69}$ have guidelines that indicate that home birth should be offered to low-risk women only. While guidelines may vary in their definition of low risk (eg, a previous cesarean section), consistent implementation and use of guidelines have been cited as an important aspect to enhance the safety of home birth. ${ }^{66}$

Evidence suggests that home birth is cost effective, in many cases significantly less expensive than hospital birth. In the UK, costs associated with low-risk vaginal birth were $50 \%$ lower in a home birth setting than in the hospital. ${ }^{70}$ However, in many areas, it is not part of the established maternity payment system (national health care or private insurance), rendering home birth out of reach financially for low-income women. ${ }^{70}$ While there has been concern raised about the challenges of balancing risk, cost, and access to care in all settings, a recent policy change in the UK has been recommended that encourages women to consider the full range of options available to them for maternity care, which includes home, hospital, and birth center, as well as type of provider being midwife or physician. ${ }^{12}$

Recommendations for policy, providers, and women choosing place of birth vary widely, and most likely there will not be consensus regarding best practice for place of birth for low-risk women among maternity care providers and policy makers in developed countries in the near future. There is, however, some indication that home birth may be more accepted and in some cases even encouraged, as in the case of the recent National Institute for Health and Care Excellence guidelines from the UK that recommend that low-risk multiparous women consider staying home or giving birth in a midwifery-led birthing unit. ${ }^{12}$ This is a significant change resulting from the recent accumulation of evidence in support of the safety of home birth within the UK. ${ }^{20}$

\section{Limitations}

Because this review was limited to those resources available in English, some pertinent studies may have been excluded. Because we did not conduct a meta-analysis, we cannot speak to the combined results of the study outcomes, only to how they exemplify the outcomes of home birth in a particular country and study population. In addition, the limitations of specific studies were addressed throughout this review, as well as the overall challenges associated with home birth-related research.

While evidence regarding neonatal outcomes related to home birth remains inconclusive, what is clear is that when guidelines and systems of transfer are in place, there is either minimal or no increased risk associated with home birth for low-risk women., ${ }^{4,620,24-34}$ Maternal outcomes are consistently in favor of planned home birth. Low-risk women experience 
less intervention and fewer complications when they plan a home birth, even if they ultimately give birth within the hospital. ${ }^{4,20,23-28,32-35,37}$ Additionally, women are very satisfied with their birth experience within the home setting. ${ }^{49,56,59}$ When not only neonatal but maternal factors are taken into consideration, there is compelling evidence that home birth should be available to low-risk women who choose it, and that policies should be in place to support integrated systems of care to support it.

\section{Disclosure}

The authors report no conflicts of interest in this work.

\section{References}

1. Office for National Statistics. Births in England and Wales by Characteristics of Birth 2, 2012. Available from: http://www.ons.gov.uk/ ons/rel/vsob1/characteristics-of-birth-2--england-and-wales/2012/ sb-characteristics-of-birth-2.html. Accessed February 13, 2015.

2. MacDorman MF, Mathews TJ, Declercq E. Trends in Out-of-Hospital Births in the United States, 1990-2012. NCHS Data Brief. 2014; No 144.

3. New Zealand Ministry of Health-Maternity Tables 2011. Available from: http://www.health.govt.nz/publication/maternity-tables-2011\#home. Accessed February 13, 2015.

4. de Jonge A, Geerts CC, van der Goes BY, Mol BW, Buitendijk SE, Nijhuis JG. Perinatal mortality and morbidity up to 28 days after birth among 743,070 low-risk planned home and hospital births: a cohort study based on three merged national perinatal databases. BJOG. 2014; Epub 2014 Sep 10.

5. Lindgren H, Radestad I, Christensson K, Hildingsson I. Outcome of planned home births in Sweden 1992-2005. Acta Obstet Gynaecol Scand. 2008;87:751-759.

6. Kataoka Y, Eto H, Iida M. Outcomes of independent midwifery attended births in birth centres and home births: a retrospective cohort study in Japan. Midwifery. 2013;29:965-972.

7. National Institute for Health and Welfare. Births and Newborns 2009. Available from: http://www.stakes.fi/FI/tilastot/aiheittain/ Lisaantyminen/synnyttajat/index.htm. Accessed February 13, 2015.

8. National Statistics Office. Statistics Canada - Births 2008 Archived Content. Available from: http://www.statcan.gc.ca/pub/84f0210x/2008000/ t026-eng.pdf. Accessed February 13, 2015.

9. Li Z, Zeki R, Hilder L, Sullivan EA. Australia's Mothers and Babies 2011. Perinatal Statistics Series 28. Available from: http://www.aihw. gov.au/WorkArea/DownloadAsset.aspx?id=60129545698. Accessed February 13, 2015.

10. Blix E, Huitfeldt AS, Øian P, Straum B, Kumle M. Outcomes of planned home births and planned hospital births in low-risk women in Norway between 1990 and 2007: a retrospective cohort study. Sex Reprod Healthcare. 2012;3:147-153.

11. Royal College of Obstetricians and Gynaecologists/Royal College of Midwives. Joint statement No 2, April 2007. Available from: https:// www.rcog.org.uk/files/rcog-corp/uploaded-files/\%20JointStatmentHomebirths2007.pdf. Accessed December 22, 2014.

12. National Institute for Health and Care Excellence. Intrapartum care: care of healthy women and their babies during childbirth. NICE clinical guideline 190 December 2014. Available from: Guidance.nice.org.uk/ cg190. Accessed February 13, 2015.

13. American College of Nurse-Midwives. Position Statement - Home Birth. Available from: www.midwife.org. Accessed February 13, 2015.

14. Midwives Alliance of North America. Home birth statement. 2012 Available from:http://mana.org/pdfs/MANAPositionStatements.pdf. Accessed December 17, 2014.
15. American College of Obstetricians and Gynecologists. Committee opinion: planned home birth. Obstet Gynecol. 2011;117(2):425-428.

16. American Academy of Pediatrics. Policy statement: planned home birth. Pediatrics. 2013;131:1016-1020.

17. Vedam S, Schummers L, Stol K, Fulton C. Home birth: an annotated guide to the literature. September 2012. Available from: http://mana. org/pdfs/HomeBirthAnnotatedGuidetotheLiteratureDec5-2012FINAL. pdf. Accessed February 13, 2015

18. Vedam S, Stoll K, Schummers L, Fulton C. Home birth: an annotated guide to the literature. October 2013. Available from: http://medfom-midwifery.sites.olt.ubc.ca/files/2014/01/HomeBirth_AnnotatedGuideToTheLiterature.pdf. Accessed January 21, 2015.

19. Hildingsson I, Lindgren H, Haglund B, Radestad I. Characteristics of women giving birth at home in Sweden: a national register study. Am J Obstet Gynecol. 2006;195(5):1366-1372.

20. Birthplace in England Collaborative Group. Perinatal and maternal outcomes by planned place of birth for healthy women with low risk pregnancies: the Birthplace in England national prospective cohort study. BMJ. 2011;343:d7400.

21. Dowswell T, Thornton JG, Hewison J, Lilford RJL. Should there be a trial of home versus hospital delivery in the United Kingdom? Measuring outcomes other than safety is feasible. BMJ. 1996;312:753-757.

22. Hendrix M, Van Hork M, Moreta D, et al. Why women do not accept randomization for place of birth: feasibility of a RCT in the Netherlands. BJOG. 2009;116:537-544.

23. Kennare RM, Keirse MR, Tucier GR, Chan AC. Planned home and hospital births in South Australia 1991-2006: differences in outcomes. MJA. 2010;192:76-80.

24. Catling-Paul C, Coddington RL, Foureur MJ, Homer CS. Publicly funded homebirth in Australia: a review of maternal and neonatal outcomes over 6 years. MJA. 2013;198(1):616-620.

25. Homer CS, Thornton C, Scarf VL, et al. Birthplace in New South Wales, Australia: an analysis of perinatal outcomes using routinely collected data. BMC Pregnancy Childbirth. 2014;14:206.

26. Hutton E, Reitsma A, Kaufman K. Outcomes associated with planned home and planned hospital births in low-risk women attended by midwives in Ontario, Canada, 2003-2006: a retrospective cohort study. Birth. 2009;36(3):180-189.

27. Janssen PA, Saxel L, Page LA, Klein MC, Liston RM, Lee SK. Outcomes of planned home birth with registered midwife versus planned hospital birth with midwife or physician. CMAJ. 2009;181(6-7):377-383.

28. Hirazumi Y, Suzuki S. Perinatal outcomes of low-risk planned home and hospital births under midwife-led care in Japan. J Obstet Gynaecol Res. 2013;39(11):1500-1504.

29. de Jong A, van der Goes BY, Ravelli ACJ, et al. Perinatal mortality and morbidity in a nationwide cohort of 529,688 low risk planned home and hospital births. BJOG. 2009;116:1177-1184.

30. van der Kooy J, Peoran J, De Graff JP, et al. Planned home compared with planned hospital births in the Netherlands: intrapartum and early neonatal death in low-risk pregnancies. Obstet Gynecol. 2011;118: 1037-1046.

31. de Jonge A, Mesman AJ, Mannien J, Zwart JJ, van Dillen J, van Roosmalen J. Severe adverse maternal outcomes among low risk women with planned home versus hospital births in the Netherlands: nationwide cohort study. BMJ. 2013;346.

32. Davis D, Baddock S, Pairman S, et al. Planned place of birth in New Zealand: does it affect mode of birth and intervention rates among low risk women? Birth. 2011;38(2):111-119.

33. Nove A, Berrington A, Matthews Z. Comparing the odds of postpartum haemorrhage in planned home birth against planned hospital birth: results of an observational study of over 500,000 maternities in the UK. BMC Pregnancy Childbirth. 2012;12:130.

34. Johnson KC, Daviss B. Outcomes of planned home births with certified professional midwives: large prospective study in North America. BMJ. 2005;330:1416-1419.

35. Grünebaum A, McCullough LB, Sapra KJ, et al. Apgar score of zero at five minutes and neonatal seizures or serious neurologic dysfunction in relation to birth setting. Am J Obstet Gynecol. 2013;209:323:e1-e6. 
36. Cheng YW, Snowden JM, King TL, et al. Selected perinatal outcomes associated with planned home births in the United States. Am J Obstet Gynecol. 2013;209(4):325e1-325e8.

37. Cox KJ, Schlegel R, Payne P, Teaf D, Albers L. Outcomes of planned home births attended by certified nurse-midwives in Southeastern Pennsylvania, 1983-2008. J Midwifery Womens Health. 2013;58: 145-149.

38. Grünebaum A, McCullough LB, Sapra KJ, et al. Early and total neonatal mortality in relation to birth setting in the United States 2006-2009. Am J Obstet Gynecol. 2014;211:390.e1-e7.

39. Grünebaum A, McCullough LB, Brent RL, Arabin B, Leven MI, Chervenak FA. Perinatal risks of planned home births in the United States. Am J Obstet Gynecol. Epub 2014 Oct 15.

40. Cheyney M, Bovbjerg M, Everson C, Gordon W, Hannibal D, Vedam S. Outcomes of care for 16,924 planned home births in the United States: the Midwives Alliance of North America statistics project, 2004 to 2009. J Midwifery Womens Health. 2014;59(1):17-27.

41. Collaborative survey of perinatal loss in planned and unplanned home births. Northern Region Perinatal Mortality Survey Coordinating Group. BMJ. 1996;313(7068):1306-1309.

42. Ehrenstein V. Association of Apgar scores with death and neurologic disability. Clin Epidemiol. 2009;1:45-53.

43. Zollinger TW, Przbylski MJ, Gamache RE. Reliability of Indiana birth certificate data compared to medical records. Ann Epidemiol. 2006; 16(1):1-10.

44. Cheyney M, Everson C, Burcher P. Homebirth transfers in the United States: narratives of risk, fear, and mutual accommodation. Qual Health Res. 2014;24(4):443-456.

45. Wax JR, Lee Lucas F, Lamont M, Pinette MG, Cartin A, Blackstone J. Maternal and newborn outcomes in planned home birth vs planned hospital births: a metaanalysis. Am J Obstet Gynecol. 2010;203: 243e1-243e8.

46. Keirse MJ. Home birth: gone away, gone astray, and here to stay. Birth. 2010;37(4):341-346.

47. Wiegers TA, Keirse MJ, van der Zee J, Berghs GA. Outcome of planned home and planned hospital births in low risk pregnancies: prospective study in midwifery practices in the Netherlands. BMJ. 1996; 313(7068):1309-1313.

48. American College of Obstetricians and Gynecologists. Safe prevention of the primary cesarean delivery. Obstetric Care Consensus No 1. Obstet Gynecol. 2014;123:693-711.

49. Bernhard C, Zielinski R, Ackerson K, English J. Home birth after hospital birth: women's choices and reflections. J Midwifery Womens Health. 2014;59(2):160-166.

50. Boucher D, Bennett C, McFarlin B, Freeze R. Staying home to give birth: why women in the United States choose home birth. J Midwifery Womens Health. 2009;54(2):119-126.

51. Catling C, Dahlen H, Homer CS. The influences on women who choose publicly-funded home birth in Australia. Midwifery. 2014;30(7): 892-898.

52. Farrish J, Von Robertson R. A qualitative examination of factors that influence birthing options for African American women. Critical Sociology. 2012;40(2):271-283.

53. Hildingsson I, Radestad, Lindgren H. Birth preferences that deviate from the norm in Sweden: planned home birth versus planned cesarean section. Birth. 2010;288-295.
54. Jouhki M. Choosing homebirth: the women's perspective. Women Birth. 2012;25(4):e56-e61.

55. Lindgren H, Erlandsson K. Women's experiences of empowerment in a planned home birth: a Swedish population-based study. Birth. 2010; 37(4):309-317.

56. Lindgren H, Hildingsson I, Rådestad I. A Swedish interview study: parents' assessment of risks in home births. Midwifery. 2006;22(1): 15-22.

57. Merg AL, Carmoney P. Phenomenological experiences: homebirth after hospital birth. Int J Childbirth Ed. 2012;27:70.

58. Murray-Davis B, McNiven P, McDonald H, Malott A, Elarar L, Hutton E. Why home birth? A qualitative study exploring women's decision making about place of birth in two Canadian provinces. Midwifery. 2012;28(5):576-581.

59. Sjöblom I, Nordström B, Edberg A. A qualitative study of women's experiences of home birth in Sweden. Midwifery. 2006;22(4):348-355.

60. Niuwenhujze M, Kane Low L. Facilitating women's choice in maternity care. J Clin Ethics. 2013;24(3):276-282.

61. Chervenak FA, McCullough LB, Grünebaum A, Arabin B, Levene MI, Brent RL. Planned home birth in the United States and professionalism: a critical assessment. J Clin Ethics. 2013;24(3):184-191.

62. McCourt C, Rayment J, Rance S, Sandall J. Organizational strategies and midwives' readiness to provide care for out of hospital births: an analysis from the Birthplace organizational case studies. Midwifery. 2012;28:636-645.

63. International Confederation of Midwives Global Standards for Midwifery Education (2010) Amended 2013. Available from: http://www. internationalmidwives.org/assets/uploads/documents/CoreDocuments/ ICM\%20Standards\%20Guidelines_ammended2013.pdf. Accessed February 13, 2015.

64. Rooks J. Midwifery and Childbirth in America. Philadelphia, PA: Temple University Press; 1996.

65. Blix E, Kumle M, Kjaergaard H, Øian P, Lindgren H. Transfer to hospital in planned home births: a systematic review. BMC Pregnancy Childbirth. 2014;14:179.

66. Cook D, Avery M, Frisvold M. Formulating evidence-based guidelines for certified nurse-midwives and certified midwives attending home births. J Midwifery Womens Health. 2014;59:153-159.

67. Obstetric Working Group. Obstetric Manual: Final Report of the Obstetric Working Group of the National Health Insurance Board of the Netherlands. The List of Obstetric Indications. Amstelveen, Netherlands: Royal Dutch Association of Midwives; 2010.

68. National Institute for Health and Clinical Excellence. Clinical Guideline No 55: Care of Healthy Women and Their Babies During Childbirth. London, UK: National Institute for Health and Clinical Excellence; 2007.

69. New Zealand Ministry of Health. Guidelines for Consultation with Obstetric and Related Medical Services (Referral Guidelines). Wellington, New Zealand: Ministry of Health; 2012.

70. Schroeder E, Petrou S, Patel N, et al. Cost effectiveness of alternative planned places of birth in women at low risk of complications: evidence from the Birthplace in England national prospective cohort study. BMJ. 2012;344:e2292.
International Journal of Women's Health

\section{Publish your work in this journal}

The International Journal of Women's Health is an international, peerreviewed open-access journal publishing original research, reports, editorials, reviews and commentaries on all aspects of women's healthcare including gynecology, obstetrics, and breast cancer. The manuscript management system is completely online and includes

\section{Dovepress}

a very quick and fair peer-review system, which is all easy to use. Visit http://www.dovepress.com/testimonials.php to read real quotes from published authors. 